\title{
The Role of Procalcitonin in the Early Diagnosis of Septic and Non-Septic Complications in the Immediate Postoperative Setting After Orthotopic Liver Transplantation
}

\author{
Aristotelis Perrakis, Volker Müller, Werner Hohenberger and Roland S. Croner \\ Additional information is available at the end of the chapter
}

http://dx.doi.org/10.5772/50242

\section{Introduction}

Very good survival rates have been achieved in patients with end stage liver disease after orthotopic liver transplantation (OLT), but in the MELD (model of end stage liver disease) era, the gap between patients in the need of OLT and available grafts has widened, leading to acceptance of marginal organs. This is one of the most important reasons for a complicated postoperative course, having a greater risk of graft or multiorgan dysfunction and systemic infection. The result of these factors is the increase of posttransplant morbidity and mortality [1].

Sepsis is the most common cause of mortality, especially in patients undergoing an immunsuppressive therapy and in these after a major surgical treatment. Despite the development and administration of new antimicrobial therapeutic modalities, the mortality rate of sepsis remains high over the last decades because of the high co morbidity and often because of delayed establishment of the diagnosis and treatment [1-4].

\subsection{Sepsis and systemic inflammatory response syndrom}

Sepsis and systemic inflammation response syndrome (SIRS) induce rapid and profound changes in endothelial function. The endothelial cells from their side trigger the immune response and activate the coagulation system. Endothelial cells cover the surface of blood vessels and are directly in tight contact with solid organs. This is the reason, why endothelial activation and damage leads to organ dysfunction. The endothelium plays also an important role in local and systemic immune responses; it is considered both as source of 
and as target for inflammation [5]. In cases of local activation of the endothelium there is an isolation of infectious procedures. On the other side and in cases of systemic activation the results are capillary leakage, hypotension and microvascular thrombosis. These aspects result in tissue hypoxia, and organ dysfunction [6-10].

In cases of inflammation, systemic infection and sepsis there is a constant exposure of these endothelial cells to the circulating endotoxins and proinflammatory cytokines such as interleucin 6 (IL-6), interleucin 1 (IL-1), interleucin 8 (IL-8), tumor necrosis factor $\alpha$ (TNF $\alpha$ ) and this leads to an activation of the endothelium and expression of endothelial leucocyte adhesion molecule (ELAM-1), intercellular adhesion molecule (ICAM-1) and vascular cell adhesion molecule (VCAM-1, 8). In cases of severe systemic inflammation and sepsis there is a significant level increase of these adhesion molecules and they could have a very good prognostic and diagnostic power, on the other side with a low specificity as far as the origin of the inflammation is concerned (infectious, non-infectious).

\subsection{Markers for sepsis}

Markers of sepsis and endothelial activation could potentially play a role in prediction and early diagnosis of onset and severity of the systemic inflammation and through differentiation between infectious and non-infectious causes of the SIRS.

The endothelial adhesion molecules (ELAM-1, ICAM-1 and VCAM1) are not sufficiently investigated and due to lack of specificity do not play a role in the clinical area.

A milestone in the sepsis era was the introduction of IL-6. IL-6, produced by various cell types, such as macrophages, monocytes and endothelial cells, can be stimulated be through bacteria, viruses, polysaccharides, TNF- $\alpha$, interferons (especially interferon $\gamma$ ) and platelet derivated growth factor (PDGF). Through its very high sensitivity there was a reasonable euphoria, as many thought that the "ideal sepsis marker" was found. However and after introduction of the study of Habarth et al [11,12], who examined combined the diagnostic relevance of IL-6 and PCT in terms of sepsis, it was shown that the diagnostic accuracy of IL-6 is impaired through inflammation procedures independent of infection. On the other hand PCT seemed to have a better correlation to septic episodes and infection.

\subsection{Sepsis and SIRS in hepatic dysfunction and after OLT}

Liver transplant recipients often manifest a systemic inflammatory response syndrome (SIRS), because of various surgical and non surgical procedures, such as induction of the immunsuppressive therapy and reperfusion during transplantation, independently of the presence of an infectious focus. The clinical signs of infection and routine laboratory tests are not specific and are sometimes misleading. The lack of specific early markers of infection might be responsible in part for delaying of a targeted antimicrobial treatment in this patient population. Moreover, the uncontrolled use of wide spectrum antimicrobial agents as "prophylactic therapy" without use of terms of the pre-emptive therapy is leading to 
bacterial translocation and the presence of multiresistant microorganisms, which further impairs the patients' outcome [13-20].

Patients with impaired hepatic function are susceptible to an infectious episode because of immunologic deficits in the period before OLT. Impaired immunologic mechanisms resulting from the hepatic function deficit such as decreased hepatic production of complement, impaired Kupffer cell function, impairment of neutrophil chemotaxis, and of inflammatory cytokines [15-20]. Due to the immunological deficit there is also a decreased activity of immunoglobulins as answer to the circulation of gram-negative pathogens [20]. In the presence of structural and functional impairment of the liver, there is a damage of filtering by impaired macrophage mobilization and diminished killing of bacteria [20]. These described deficits lead to an increased risk for systemic infection in the early posttransplant setting and this fact is the most frequent and dangerous complication occurring after OLT. Mora et al, Singh et al and Lee et al reported in their studies the relevance of the early and late bacteriemia in liver transplant recipients, the higher mortality rates in comparison to other patient populations, such as surgical patients and the great importance of the early diagnosis and the early onset of a targeted antimicrobial therapy in order to achieve an acceptable posttransplant outcome [21-24]. Kim et al. reported also similar risk factors in their study about bacteriemia in living donor liver transplant recipients (LDLT) [25]. Several authors reported in their studies an increased infection risk in LDLT because of the small graft size, which might lead to an increased portal venous pressure with impaired bowel motility with consecutive bacterial translocation, which increases the risk for a bacterial infection [26,27]. Igekami et al reported in a large study with LDLT recipients that late enteral nutrition and great blood loss during operative procedure were significant and independent risk factors for a bacterial sepsis in the posttransplant setting [28]. The early start of enteral nutrition could be effective in transplant patients, because of the stimulating effect in terms of bile flow, the enterohepatic circulation and portal blood flow, preventing an atrophia of the intestinal mucosa and a bacterial translocation [28]. Furthermore high MELD score and the co morbidity of recipients seem to have a significant effect on the onset of bacteriemia and septic complications after OLT.

Bacteriemia accounts for up to $30 \%$ of all major infections after liver transplantation with an considerable mortality risk of $20 \%$ to $39 \%$ [21-28]. The main sources for bacteriemia and systemic infection, occurring within the first 3 months after OLT, are infected vascular catheter sited, pneumonia and infections of the biliary tract in terms of cholangitis following a biliary disorder( stenosis, ITBL, presence of biliodigestive anastomosis) [29]. Several authors examining blood stream infections in the posttransplant setting in their studies have shown that gram negatives, particularly, Klebsiella and Pseudomonas, tend to be the predominant species [30]. Furthermore Karvellas et al reported in a large study that the predominant species was enterococcus. Significantly more patients in the infected group had a biliodigestve anastomosis [30].

As underlined before, several studies have examined the risk factors and the onset of posttransplantation bacterial and fungal sepsis in patients undergoing OLT. Clinical signs of an 
infection (dysregulation of temperature, tachycardia) are either absent or nonspecific $[4,13$ 31-44]. Various authors underlined the great importance of early initiation of a targeted antimicrobial therapy or the preemptive therapy in high-risk patients in order to achieve acceptable outcomes by preventing generalized sepsis, septic shock and multiorgan dysfunction.

Especially bacterial sepsis has a significant impact on the survival of patients who undergo a transplant proceeding because of the surgical trauma and the need for immunosuppression [1-4].

Furthermore, commonly used biochemical markers, such as C-reactive protein (CRP) or leucocyte count, cannot be considered to be specific inflammation markers in the early postoperative setting in order to establish a definite diagnosis [42].

\section{Body}

\subsection{Procalcitonin; background}

Calcitonin, a 32-amino-acid amidated hormone, is initially biosynthesized as a precursor, named preprocalcitonin [45] This protein is processed into several calcitonin precursors. In adults and children, infection and sepsis have been found to be associated with increased serum levels of calcitonin precursors [46-53]. These concentrations were shown to be positively correlated with a subsequent mortality rate [45]. Circulating calcitonin precursors, including procalcitonin, are raised in severe bacterial infections, but remain in low or normal ranges in viral infections and non-specific inflammatory diseases. $(38,45,46)$.

Procalcitonin (PCT) is a precursor protein of the hormone calcitonin with a molecular weight of approximately $13 \mathrm{kDa}$. PCT is induced in the plasma of patients with severe bacterial or fungal infections or sepsis [38,40-54]. Local bacterial infections, viral infections, autoimmune and allergic disorders do not induce PCT. At present, it is not clear whether PCT is predominantly influenced merely by inflammation induced by microbial infections, or also by the severity of multiple organ dysfunctions secondary to the systemic inflammatory response.

Procalcitonin (PCT) is identified as a diagnostic marker for infectious or septic processes and correlates better with its severity as CRP [5, 44-57]. PCT is an acute phase protein, composed of 116 amino acids and is the precursor of calcitonin. In healthy individuals, PCT serum concentrations are very low $(<0,5 \mathrm{mg} / \mathrm{dl})$. The half-life of PCT is approximately 26 to 30 hours [38-40]. Neuroendocrine cells of solid organs (lung, kidney, pancreas, adrenal gland, and liver) might be the source of PCT during an inflammatory procedure. A further role might play extrathyroid cells, such macrophages, monocytes and liver cells in the PCT production during sepsis [40,41]. The main hypothesis as far as the PCT induction is concerned, presents that the stimulation for PCT elevation is a result of a systemic challenge of the organism with bacterial endotoxin and bacterial polysaccharides [39]. Overall the exact pathophysiology is still an open matter. $[38,40]$. 


\subsection{PCT after liver surgery and in hepatic dysfunction}

Procalcitonin (PCT) is a well established marker indicating a complication such as inflammation or sepsis among surgical patients. PCT rises $24-48$ hours before and correlates better with the severity of sepsis and infection than CRP. In uncomplicated liver surgery cases, PCT peaks at 24 hours after the Pringle maneuver (clamp of the hepatoduodenal ligament) with a half-life of 24-30 hours. It has been reported that the liver has a primary role as the source of PCT production during endotoxin shock [24] In cirrhotic patients with impaired hepatic synthetic function, PCT levels were not lower, displaying the same predictive power for infection as in patients without cirrhosis [44].

\subsection{PCT in trauma and surgery}

Meissner et al emphasizing the differences in the kinetics of the main inflammation markers (PCT, CRP) after trauma and surgery, reported in their study that unlike CRP, PCT values typically declined rapidly after trauma. The rapid decline of the initial induction of PCT after trauma or surgery compared with the long-lasting increase of CRP promises an earlier use of PCT as a marker of sepsis and infection than of CRP in the postoperative /posttraumatic phase. He also demonstrates that the CRP level in trauma patients is not a valid parameter to gather more information about the severity of systemic inflammation, complications, and prognosis of the patient. PCT and clinical score systems are superior to CRP for risk stratification of the patient. On the contrary, the diagnostic use of CRP after trauma is limited because of its slow kinetics [35, 37, 39, 54, 55]. Other studies emphasize the major role of early changes of PCT levels for predicting the final outcome of septic patients. Any decrease of serum PCT within the first 48 hours by $30 \%$ or its persistence at levels within the normal ranges was an indicator of favorable outcome. On the contrary, any increase of serum PCT after 48 hours or any minimal decrease is a sign for insufficient antimicrobial therapeutic strategy. This finding applied both for patients with sepsis and for patients with severe sepsis/shock. It was a factor related to final outcome independently from disease severity.

\subsection{PCT in OLT}

However, the prognostic value of procalcitonin in liver transplant recipients, undergoing immunsuppression and showing severe co morbidities remains to be determined. Initially high PCT levels do not necessarily indicate a poor prognosis as do continuous or secondarily rising levels or elevations in the later postoperative course [42, 43]. In the posttransplant setting, elevated PCT levels are observed in the first 2-3 days after OLT [1]. Initially high PCT levels, however, do not necessarily indicate a poor prognosis as do continuous or secondarily rising levels [32,42]. Cardiac arrest and infection, but not PCT level in the donor, are associated with high post- OLT PCT levels in the recipient [43]. In patients with impaired hepatic synthesis, PCT levels were not lower; displaying the same predictive relevance for infection as in septic patients without cirrhosis or impairment of the 
liver function [44]. PCT has been shown to increase among OLT patients with infections, but it fails to predict an acute rejection episode. Furthermore, PCT rises in all patients undergoing OLT owing to the perioperative distress.

Coelho et al reported in a study with pediatric liver transplant recipients that it was possible to differentiate between bacterial infection and rejection by PCT measurement. In all patients with bacterial infection, an increase in PCT was to register. On the other hand patients suffering from acute graft rejection and these having an uneventful posttransplant course did not show an increase of PCT [56].

Furthermore an ATG (antithymocyte globulin)-based immunosuppressive therapy is a stimulus for synthesis and further elevation of PCT [53]. This fact presents the major limitation of PCT's diagnostic power. Additionally the presence of viral infection did not stimulate a PCT elevation and cannot be diagnosed through this inflammation marker [32,37-60].

Van den Broek et al [32] in a large study with 135 patients (LDLT and deceased donor recipients) examined the diagnostic and prognostic accuracy of PCT in cases of critical systemic infections in comparison to other traditional inflammation markes such as CRP and leucocyte count (LC). In this study peak PCT was found not to be an independent factor for occurrence of serious infections and septic episodes. On the other hand the authors underlined the importance of CRP, as it was found to be an independent risk factor for a critical systemic infection. Eyraud et al [43] investigated also the relevance of PCT in the posttransplant setting after OLT. The results of this study could not confirm them of previous studies [58,59] reporting that peak PCT could have its origin either from an infection of the recipient or from cardiac arrest of the donor. In this study the clinical characteristics of the donor are emphasized: the initial PCT of recipient has its origin from donor's cardiac arrest and it could not be predictive of graft dysfunction or postoperative complications after OLT. Furthermore graft preservation, graft flushing and type of reperfusion are factors, which have a significant influence on initial PCT values and this is one of the main bias as far as the sensitivity of PCT in the early posttransplant phase is concerned. Therefore Fazakas et al proposed standard procedures for graft harvesting, timing and technique of graft flushing in order to avoid the pathophysiological conditions, which lead to this bias [59].

The most significant value as far as the diagnostic power of PCT is concerned is the fact, that PCT levels increase far less than CRP levels, and the period of unspecific induction is much shorter [54].

\subsection{PCT in MODS}

In another large study with critically ill patients Meissner and colleagues indicate that PCT concentrations are associated with the severity of multiorgan dysfunction (MODS) during sepsis as assessed by the SOFA (sepsis-related organ failure assessment) score [39]. PCT has several advantages in severely ill patients compared with CRP. The most striking one, 
demonstrated in his study, is the enormous range of PCT reactivity resulting in a marked increase in PCT plasma levels, especially during severe stages of MODS and systemic inflammation. On the other hand, PCT concentrations are quite low when only a moderate organ dysfunction or a weak systemic inflammatory response is present. In contrast, CRP levels are often found to be already increased to maximal concentrations in patients with low SOFA scores. Thus, CRP cannot provide information as to further increases in organ dysfunction and the inflammatory progress, respectively, since it is already increased to its maximum values during a less severe stage of disease. Further advantages of PCT are its more rapid kinetics; PCT reacts faster than CRP both during an increase or decrease of inflammation. Regarding the prognosis of the disease, the course of PCT after day 4 from the onset of systemic inflammation was able to distinguish survivors from non-survivors. In summary, PCT compared with CRP is characterized by its ability to be induced to very high serum concentrations also during advanced stages of MODS and severe systemic inflammation, respectively, whereas CRP is often already in the upper concentration range, even in patients with low severity scores, and exhibits no such further dynamics during the course of MODS and systemic inflammation. PCT more rapidly declines to the normal range during the recovery of the patient compared with CRP, and thus provides more information in patients with MODS and sepsis of various etiologies than CRP.

Therefore the examination and the careful evaluation of postoperative PCT values are the most important stages for the early diagnosis of sepsis and infection after surgery.

Various authors and we emphasize the fact that the diagnostic accuracy of procalcitonin is completely dependent on use of a sensitive assay in an appropriate clinical setting. The clinical signs, physical examination and the clinical setting cannot be substituted by procalcitonin. They must be seen as complementary tools for early establishment of diagnosis. [38,42]

\subsection{PCT: Our observations during liver transplantation}

Aim of our research was to evaluate PCT as an early prognostic marker within 48 hours after OLT for subsequent postoperative complications and to assess the prognostic accuracy of PCT as marker for infectious and non infectious postoperative complications, septic episodes and as predict factor as far as the in hospital mortality is concerned. Our research included a retrospective study in a cohort liver transplant recipients underwent 32 liver transplantations [42] and a prospective part including patients underwent 75 OLT.

Patients with irreversible end-stage liver disease that was life threatening and refractory to other forms of conventional medical or surgical therapy who had no contraindications for transplantation were regarded as eligible for OLT. The organs were allocated according to the Model of End Stage Disease (MELD) [61]

For each recipient, the following were recorded: age, gender and PCT serum concentration before, 6 hours after reperfusion and then daily during the stay in the ICU after OLT. 
Blood samples were obtained for routine testing (biochemical parameters), and for each patient, serum aliquots were used for PCT determination. The procalcitonin levels were measured by an immunoluminometric LUMItest PCT kit (Brahms; Diagnostica, Berlin, Germany) ; the normal range detected was from 0.1 to $0.5 \mathrm{ng} / \mathrm{mL}$.

Postoperative clinical course was prospectively analyzed from admission to discharge according to main clinical data: infectious/non infectious complications, septic episodes, graft disorders, acute rejection episodes, renal failure, multiorgan failure and need for retransplantation. Complications were graded according to the classification of Dindo et al [62].

\subsubsection{Definitions for our protocol}

Graft dysfunction was defined as the occurrence of at least one of the following criteria: the need for retransplantation (primary non function, PNF), a rise in aminotransferases of above $2,000 \mathrm{UI} / \mathrm{L}$, impairment of factor $\mathrm{V}(<30 \%)$ with synchronous increase of bilirubin without a retrospective need for retransplantation, serum bilirubin greater than $10 \mathrm{mg} / \mathrm{ml}$; PT of at least $17 \mathrm{sec}$; hepatic encephalopathy [63]. Mortality was defined as death from any cause occurring during the hospital stay.

Pulmonary complication was defined as the need for mechanical ventilation.

Acute renal failure was defined as plasma creatinemia of greater than $2 \mathrm{mg} / \mathrm{dl}$ and urine output of less than $0.5 \mathrm{~mL} /$ hour. Renal complication was defined as the need for dialysis after OLT or greater than $100 \%$ of creatinine levels compared with preoperative values.

Postoperative complication was defined as hepatic dysfunction, infection, or pulmonary or renal complication or other surgical complication such as bleeding, hepatic artery thrombosis (HAT), thrombosis of the portal or cava vein and biliary complication such as insufficiency, stenosis, ITBL(ischemic type biliary lesion).

The definition of clinical infection was standardized with criteria proposed by the Centers for Disease Control and Prevention and included pulmonary, bloodstream, or intraabdominal infections accompanied by clinical symptoms proven by microbiological, radiological, or surgical findings and reacting to instituted therapy.

Infection was diagnosed if clinical; biochemical or radiologic signs of infection were evident. Chest X-rays, ultrasound examinations were performed on daily basis during the ICU stay and computed tomography (CT) scans when a clinical infection was suspected, and on the basis of these examinations, prompt therapy was initiated. In cases where samples from the suspected site of infection were positive, a proven infection could be defined.

Sepsis was defined by the presence of infection and SIRS (systemic inflammatory response syndrome). The criteria for SIRS were met if more than one of the following signs occurred: a body temperature greater than $38^{\circ} \mathrm{C}$ or less than $36^{\circ} \mathrm{C}$, a heart rate greater than 100 beats per minute, hyperventilation as evidenced by a respiratory rate greater than 20 breaths per minute or a partial pressure of carbon dioxide in the arterial blood less than $32 \mathrm{~mm} \mathrm{Hg}$, and 
an LC greater than 10.000 cells/ $\mu$ l or less than 4.000 cells/ $\mu 1.5$ Severe sepsis was defined as sepsis complicated by organ dysfunction [64]

\subsubsection{Immunsuppression protocol and antibiotic prophylaxis}

All patients were initially treated with tacrolimus, starting 36 hours after transplantation at $0.1 \mathrm{mg} / \mathrm{kg}$ twice daily and $500 \mathrm{mg}$ methylprednisolone in the anhepatic phase. All patients received basiliximab (Simulect, $20 \mathrm{mg}$ ) in the anhepatic phase followed by a second administration (20mg), 4 days after transplantation. Mycophenolate mofetil (500 mg twice daily, intravenous or per oral) was administered starting on the $5^{\text {th }}$ postoperative day. Acute rejection was diagnosed based on the histopathological examination after liver biopsy according to BANFF criteria [65]. In patients with tacrolimus neurotoxicity and other Tacrolimus triggered side effects, a conversion of the immunsuppressive regimen to Ciclosporin- based immunsuppression was performed. All recipients received broadspectrum antimicrobial prophylaxis, consisting of antibacterial, antiviral, and antimycotic agents: with piperacilline-tazobactam for 7 days aciclovir and anidulafungin/posaconazole. Selective digestive decontamination consisted of $200 \mathrm{mg}$ of oral amphotericin B 3 times daily until the $21^{\text {st }}$ postoperative day. Furthermore, high-risk patients, recipients of a cytomegalovirus (CMV) - positive donor received preemptive antiviral treatment with ganciclovir/ valganciclovir adjusted to renal function over a 6-week course. The standard laboratory workup included hematologic and biochemical parameters. The CMV status (viral load, pp65 antigen) was examined twice a week. Cytomegalovirus (CMV) infection was defined by the appearance of CMV antigen polymerase chain reaction in the blood. XRay examinations of the chest and ultrasounds of the graft were performed daily.

\subsubsection{Liver transplantation}

The donor organ was always retrieved as part of a multiorgan donation. Either University of Wisconsin (UW) or histidine-tryptophan-Ketoglutarate (HTK) solution was used for preservation. Liver transplantation was performed by using either the retrocaval resection technique or the piggyback technique. We performed either simultaneous portoarterial reperfusion or a portal reperfusion of the graft in a ratio 1:1. All patients were indicated for OLT because of end stage liver disease. Indications for OLT were: 1. acute liver failure, 2. hepatocellular carcinoma inside the Milan criteria, 3. hepatitis C induced liver cirrhosis, 4 . hepatitis B induced liver cirrhosis , 5. nutritive toxic liver cirrhosis , 6. hemochromatosis , 7. autoimmunhepatitis. The median MELD score was 33 and 33 OLT were performed after acceptance of a request for high urgency $(\mathrm{HU})$ liver transplantation

\subsubsection{Statistics and results}

Linear regression was used in univariate analysis to identify predictors of elevated PCT concentrations. Multivariate analysis was carried out using a logistic regression model. All of the tests performed were two-sided. Significant predictors in univariate analysis were 
included in a multivariate linear regression model, with a stepwise variable selection method. Potential associations between infectious complications or overall complications and surgical, clinical or biological parameters were tested with univariate procedures, using students' paired t-test for continuous variables and Pearson's chi-square test for categorical variables. P-values of less than 0.05 were considered to be statistically significant.

Statistical analysis was done with SPSS (SPSS v19.0, Chicago, IL, USA).

In the retrospective part of our research 32 adult patients with end-stage liver disease underwent OLT.

In 29 patients the peak-PCT occurred within 48 hours after OLT. A rapidly rising PCT was observed in 2 patients associated with subsequent fatal outcomes. Among the 10 patients with peak-PCT values less than $5 \mathrm{ng} / \mathrm{mL}, 9$ displayed uneventful postoperative courses. PCT values more than $5 \mathrm{ng} / \mathrm{mL}$ were observed among 22 patients with 23 OLT, including 12 with 13 OLT who suffered more than 1 complications. The odds ratio (OR) for a complicated course in the presence of an initial PCT more than $5 \mathrm{ng} / \mathrm{mL}$ was calculated to be 11.7 (95\% Confidence interval, 11.3-12.1; $P<.025)$ with risk ratio of 5.65. The risk of renal failure requiring hemofiltration was independent of a PCT more than $5 \mathrm{ng} / \mathrm{mL}$ (OR 8.25; $P<0.04$ ). Total bilirubin was significantly higher among patients with an initial PCT more than 5 $\mathrm{ng} / \mathrm{mL}$ until day 7 , compared with patients with a PCT less than $5 \mathrm{ng} / \mathrm{mL}$. GOT was significantly higher on days 2 and 3 in these groups, but peak values for GOT and total bilirubin correlated significantly on univariate analysis. CRP did not show any significant difference between the complication and non-complication groups. The immunosuppression regimen was not significantly different regarding the occurrence of a complication or the occurrence of a peak-PCT of $5 \mathrm{ng} / \mathrm{mL}$.

In the prospective part of our research patients with end-stage liver disease patients underwent 75 OLT. In this part we examined the clinical significance of the occurrence of a 2nd PCT peak, after the initial PCT peak, which occurs in the immediate post transplant setting.

In 65 patients the initial peak-PCT occurred within 72 hours after OLT. Patients with initial PCT values $>5 \mathrm{ng} / \mathrm{mL}$ had a significantly higher risk to suffer more than 1 complications (infectious, non-infectious).

Overall 42 complications (infectious, non-infectious) were registered and there was a significant relevance with the occurrence of a $2^{\text {nd }}$ PCT peak to register. In patients with a graft dysfunction, including acute rejection episodes, there was a significant PCT elevation with the occurrence of a $2^{\text {nd }}$ peak. However in some patients there was a coincidence of an infectious focus to register. This fact was without statistical significance Among patients with a PNF and the need of re-transplantation, there was a significant elevation of PCT with the occurrence of a $2^{\text {nd }}$ peak. On the contrary in patients with a HAT a $2^{\text {nd }}$ PCT peak could not be registered.

Patients with biliary complications did not show an elevation of PCT or a $2^{\text {nd }}$ peak. Patients which displayed an acute renal failure after OLT with the intermittent need for CVVH 
(continuous veno-venous hemofiltration), but there was no relevance between this complication and the PCT course. Furthermore the risk of renal failure requiring hemofiltration was independent of an initial PCT $>5 \mathrm{ng} / \mathrm{mL}$

Furthermore we register that there was no relevance between the $1^{\text {st }}$ peak-PCT and the further postoperative course or the occurrence of complications (infectious or noninfectious, $\mathrm{p}=0.442$ ).

Patients, in which a $2^{\text {nd }}$ PCT peak was occurred, had a significantly higher risk for a complicated course, for a complicated sepsis course with multiorgan dysfunction, for a non infectious complication $(\mathrm{p}<0.0001)$ and for in-hospital mortality $(\mathrm{p}<0.0001)$.

\subsubsection{Interpretation of our findings}

However, the peak value of PCT, usually occurring 2nd or 3rd postoperative day, was not an independent factor for a fatal outcome according to the present series. An initially high PCT has been described to not indicate a poor prognosis when followed by an adequate decline. A rapidly rising PCT without a decline after the 3rd postoperative day is associated with a fatal outcome correlating with a bacterial or fungal infection. In contrast to earlier studies where an elevation of PCT was seen only in infectious complications, we also observed a greater number of noninfectious complications. The high PCT did not predict the type or severity of the complication in general practice, but among transplant patients a further elevation was seen only in bacterial infections and not in rejection episodes or local inflammation. We observed a rise of PCT in respiratory failure and sepsis, but not in occurrence of ascites, pleural effusion, acute rejection episodes, or bleeding, as reported by other authors.

An initially high PCT has been described not to indicate a poor prognosis. Furthermore it is generally accepted that ischemic times as well as reperfusion produce a proinflammatory response of impaired liver function. Owing to tissue damage which predisposes to generalized infection and postoperative sepsis. In addition, elevated PCT values did not suggest a graft rejection process.

Furthermore our results show that OLT recipients with infectious and some non infectious complications had significantly higher values of PCT, in the majority of cases with a $2^{\text {nd }}$ peak value after the initial PCT peak, in comparison to recipients with an uncomplicated post transplant course. The $1^{\text {st }}$ peak PCT was not an independent risk factor in LTx recipients, while the occurrence of a $2^{\text {nd }}$ peak was a significant independent factor for protracted septic course, for any complication overall (infectious or non infectious) and for post transplant mortality. The fact that the $1^{\text {st }}$ PCT peak does not play a diagnostic and prognostic role underlined also Eyraud et al [43]. In this study was stated that PCT level in the donor and early PCT peak in the recipient have not a predictive value as far as post-OLT hepatic dysfunction or other complications are concerned. Cardiac arrest and infection in the donor seem to be associated with high post- OLT PCT levels in the recipient. As underlined before 
a major limitation as far as the diagnostic accuracy of PCT is concerned stated Zazula et al, who reported the ATG-stimulated elevation of PCT [53]. This aspect was not a limitation of our research, as we prefer ATG-free immunsuppressive regimens.

The occurrence of a $2^{\text {nd }}$ PCT peak and of an eventful post transplant setting was significant in patients with a severe sepsis, bacterial or fungal infection and graft dysfunction overall (PNF and AR). In AR alone there was not a prognostic value of PCT to register. What this matter is concerned our results confirmed our first reports, as far the relevance between the $1^{\text {st }}$ PCT peak and the AR is concerned [42].

In contrast to earlier studies where an elevation of PCT was seen only in infectious complications we also observed a greater number of non infectious complications when the PCT was elevated or showed a $2^{\text {nd }}$ peak.

Our research included an acceptable cohort of deceased donor OLT recipients and showed that PCT was an independent risk factor for infectious and some non-infectious complications and that the occurrence of a $2^{\text {nd }}$ peak was an independent factor for a complicated postoperative outcome and for mortality. Furthermore we registered a high prognostic value, due to its occurrence before clinical onset of the complications and clinical impairment of the patients.

Various studies have demonstrated that PCT is a useful diagnostic marker for bacterial and fungal infections and sepsis in different patient groups with superior diagnostic accuracy in comparison with CRP $[34,44,55,57]$. Furthermore PCT was described in some small cohort studies as an accurate marker for any complication in the post transplant setting $[57,58,60]$, which could not be fully confirmed by other study groups and by the present series $[32,42,43]$.

However this research reports the significance of the role of PCT as independent factor as far as its course and the $2^{\text {nd }}$ peak are concerned. We have to admit that PCT must not be regarded to be the only reliable diagnostic parameter. Postoperative complications after liver transplantation may result from multifactorial processes [66,67], which cannot be monitored by only 1 marker.

\section{Conclusions}

OLT is a complex procedure with a great and very important physiological and pathophysiological background and many parameters and biochemical pathways, which have to be taken into consideration after the operating procedure. The most common complication after OLT is the occurrence of systemic infection and sepsis. The great operative stress, the immunsupression and other parameters, such as the reperfusion injury of the graft lead to a higher risk for occurrence of a septic complication with high morbidity and mortality rates. Therefore the sufficient monitoring of this patient population, the early diagnosis and the immediate treatment of an infectious complication are of great importance. During the last years several studies and research have been performed in 
order to find the "ideal infectious marker" with high sensitivity and specificity for the early establishment of an infectious complication. Common inflammation markers such as LC and CRP cannot be considered as sufficient markers in order to diagnose quickly an infectious complication in the posttransplant setting. IL-6 has advantages and disadvantages and is not enough investigated, especially in the clinical area and the endothelial adhesion molecules (ICAM-1, VCAM-1, ECAM-1) have not been proofed. PCT is nowadays a well established sepsis marker. However PCT must not be regarded to be the only reliable diagnostic parameter. Postoperative complications after liver transplantation may result from multifactorial processes, which cannot be monitored by only 1 marker. However, the values and especially the trend after the peak value of PCT seem to be important diagnostic tools to detect serious infectious complications. Based on our results we believe that the value of PCT and of the occurrence of a $2^{\text {nd }}$ peak seem to be important diagnostic tools for the early detection of serious infectious complications. Of great importance is to examine the correlation between PCT course, clinical course and CRP. This must be regarded as the basis of the post transplant management of a liver recipient. Furthermore our clinical research revealed that patients experienced great benefit from prophylactic and preemptive antibacterial, antifungal, and antiviral therapy.

The relevance and the importance of PCT and its course during the post transplant setting should be further examined in randomized controlled studies on multicenter basis. Furthermore we believe that inflammation markers such as IL-6 should be further examined e.g. in combination with PCT, because IL-6 is an extremely sensitive marker and could fill the "diagnostic gap" of PCT as far as viral infections are concerned. Endothelial adhesion molecules, which have the best sensitivity among the inflammation markers, are until today not examined and in our opinion have still a role to play.

\section{Author details}

Aristotelis Perrakis*, Volker Müller, Werner Hohenberger and Roland S. Croner

Surgical Department, Department of Hepatobiliary and Transplant Surgery,

University of Erlangen-Nuremberg, Erlangen, Germany

\section{References}

[1] Iida T, Kaido T, Yagi S et al.Posttransplant bacteremia in adult living donor liver transplant recipients. Liver Transpl. 2010 Dec;16(12):1379-85.

[2] Mora NP, Husberg BS, Gonwa TA, et al.The impact of the different severe infections on the outcome of liver transplantation. A study of 150 patients. Transpl Int 1992;5:S209S210.

[3] Singh N, Paterson DL, Gayowski T, et al.Predicting bacteremia and septic mortality in liver transplant recipients. Liver Transpl 2000;6:54-61.

${ }^{*}$ Corresponding Author 
[4] Lee SO, Kang SH, Abdel-Massih RC,et al. Spectrum of early- onset and late-onset bacteremias after liver transplantation: implications for management. Liver Transpl 2011; 17(6):733-41.

[5] Hack CE, Zeerleder S. The endothelium in sepsis: Source of and a target for inflammation. Crit Care Med 2001; 29(Suppl): S21-S27

[6] Mavrommatis AC, Theodoridis T, Orfanidou A, et al. Coagulation system and platelets are fully activated in uncomplicated sepsis. Crit Care Med 2000; 28:451-457

[7] Bernard GR, Vincent JL, Laterre PF, et al. Efficacy and safety of recombinant human activated protein C for severe sepsis. N Engl J Med 2001; 344:699-709

[8] Croner RS, Hohenberger W, Jeschke MG. Hepatic gene expression during endotoxemia. J Surg Res.2009 Jun 1;154(1):126-34

[9] Tschaikowsky K, Sagner S, Lehner N, et al. Endothelin in septic patients: Effects on cardiovascular and renal function and its relationship to proinflammatory cytokines. Crit Care Med 2000; 28:1854-1860

[10] Brauner JS, Rohde LE, Clausell N. Circulating endothelin-1 and tumor necrosis factor alpha: Early predictors of mortality in patients with septic shock. Intensive Care Med 2000; 26:305-313

[11] Reinhart K, Menges T, Gardlund B, et al. Randomized, placebo-controlled trial of the anti-tumor necrosis factor antibody fragment afelimomab in hyperinflammatory response during severe sepsis: The RAMSES Study. Crit Care Med 2001; 29:765-769

[12] Reinhart K, Wiegand-Löhnert C, Grimminger F, et al. The MAK 195F Sepsis Study Group. Assessment of the safety and efficacy of the monoclonal anti-tumor necrosis factor antibody-fragment, MAK 195F, in patients with sepsis and septic shock: A multicenter, randomized, placebo-controlled, dose-ranging study. Crit Care Med 1996; 24:733-742

[13] Castelli GP, Pognani C, Meisner M, et al. Procalcitonin and C-reactive protein during systemic inflammatory response syndrome, sepsis and organ dysfunction. Crit Care 2004;8 (4):234-42.

[14] Adams HG, Jordan C. Infections in the alcoholic. Med Clin North Am 1984;68:179-200.

[15] Wyke RJ. Problems of bacterial infection in patients with liver disease. Gut 1987;28:62341.

[16] Johnson DH, Cunha BA. Infections in cirrhosis. Infect Dis Clin North Am 2001;15:36371

[17] Campbell AC, Dronfield MW, Toghill PJ, et al. Neutrophil function in chronic liver disease. Clin Exp Immunol 1981;45:81-9.

[18] Fierer J, Finley F. Deficient bactericidal activity against Escherichia coli in patients with cirrhosis of the liver. J Clin Invest 1979;63:912.

[19] Liu YK. Effects of alcohol on granulocytes and lymphocytes. Semin Hematol 1980;17:130-6.

[20] Fortun J, Martin-Davila P, Moreno S, et al. Risk factors for invasive aspergillosis in liver transplant recipients. Liver Transpl 2002;8: 1065-70. 
[21] Singh N, Wagener MM, Obman A, et al. Bacteremias in liver transplant recipients: shift toward gram-negative bacteria as predominant pathogens. Liver Transpl 2004;10:844-9

[22] Mora NP, Husberg BS, Gonwa TA, et al. The impact of the different severe infections on the outcome of liver transplantation.A study of 150 patients. Transpl Int 1992;5:209-210.

[23] Singh N, Paterson DL, Gayowski T, et al. Predicting bacteremia and septic mortality in liver transplant recipients. Liver Transpl 2000;6:54-61.

[24] Lee SO, Kang SH, Abdel-Massih RC, et al. Spectrum of early and late-onset bacteremia after liver transplantation: Implications for management. Liver Transpl 2011;17:733-741

[25] Kim SI, Kim YJ, Jun YH, et al. Epidemiology and risk factors for bacteremia in 144 consecutive living-donor liver transplant recipients. Yonsei Med J 2009;50:112-121

[26] Kiuchi T, Onishi Y, Nakamura T. Small-for-size graft: not defined solely by being small for size. Liver Transpl 2010;16:815-817

[27] Shimada M, Ijichi $H$, Yonemura $Y$, et al. Is graft size a major risk factor in living-donor adult liver transplantation? Transpl Int 2004;17:310-316

[28] Igekami T, Shirabe K, Yoshiya S, et al. Bacterial Sepsis after LivingDonor Liver Transplantation: The Impact of Early Enteral Nutrition. J Am Coll Surg;214;3;288-295

[29] Soejima Y, Taketomi A, Yoshizumi T, et al. Feasibility of left lobe living donor liver transplantation between adults: an 8-year, single-center experience of 107 cases. Am J Transplant 2006;6:1004-1011.

[30] Karvellas CJ, McPhail M, Pink F, et al. Bloodstream infection after elective liver transplantation is associated with increased mortality in patients with cirrhosis. Journal of Critical Care ; 2011 26, 468-474

[31] Kornberg A, Grube T, Wagner T, et al. Differentiated therapy with prostaglandin E1 (alprostadil) after orthotopic liver transplantation: the usefulness of procalcitonin (PCT) and hepatic artery resistive index (RI) for the evaluation of early graft function and clinical course. Clin Chem Lab Med 2000 Nov;38(11):1177-80

[32] van den Broek M AJ, Damnik S W M.O, Winkens B, et al. Procalcitonin as a Prognostic Marker for infectious complications in Liver Transplant Recipients in an Intensive Care Unit. Liver Transpl 2010;16:402-410

[33] Saner FH, Olde Damink SW, Pavlakovic G, et al. Pulmonary and blood stream infections in adult living donor and cadaveric liver transplant patients. Transplantation 2008; 85:1564-1568.

[34] Kuse ER, Langefeld I, Jaeger K, et al. Procalcitonin in fever of unknown origin after liver transplantation: a variable to differentiate acute rejection from infection. Crit Care Med 2000;28:555-559.

[35] Meisner M, Tschaikowsky K, Hutzler A, et al. Postoperative plasma concentrations of procalcitonin after different types of surgery. Intensive Care Med 1998;24:680-684.

[36] Morgenthaler NG, Struck J, Chancerelle Y, et al. Production of procalcitonin (PCT) in non-thyroidal tissue after LPS injection. Horm Metab Res 2003, 35:290-295.

[37] Meisner M, Tschaikowsky K, Schmidt J, et al. Procalcitonin (PCT)-indications for a new diagnostic parameter of severe bacterial infection and sepsis in transplantation, 
immunosuppression and cardiac assist devices. Cardiovascular Engineering 1996, 1:6776

[38] Assicot M, Gendrel D, Carsin H, et al. High serum procalcitonin concentrations in patients with sepsis and infection. Lancet 1993;341:515-518.

[39] Meisner M, Tschaikowsky K, Palmaers T, et al. Comparison of procalcitonin (PCT) and C-reactive protein (CRP) plasma concentrations at different SOFA scores during the course of sepsis and MODS. Crit Care 1999;3(1):45-50.

[40] Arkader R, Troster EJ, Lopes MR, et al. Procalcitonin does discriminate between sepsis and systemic inflammatory response syndrome. Arch Dis Child 2006;91(2):117-20

[41] Brunkhorst FM, Heinz U, Forycki ZF. Kinetics of procalcitonin in iatrogenic sepsis. Intensive Care Med 1998, 24:888-889.

[42] Perrakis A, Yedibela S, Schellerer V, et al. Procalcitonin in the setting of complicated postoperative course after liver transplantation. Transplant Proc. 2010; 42(10):4187-90

[43] Eyraud D, Ben Ayed S, Tanguy ML, et al. Procalcitonin in liver transplantation: are high levels due to donors or recipients? Crit Care 2008;12: R85.

[44] Bota DP, Van Nuffelen M, Zakariah AN,et al. Serum levels of C-reactive protein and procalcitonin in critically ill patients with cirrhosis of the liver. J Lab Clin Med. 2005;146(6):347-51.

[45] Becker KL, Nylén ES, Cohen R, et al. Calcitonin gene family of peptides. In: Principles and Practice of Endocrinology and Metabolism. Becker KL (Ed). Second Edition. Philadelphia, JB Lippincott, 1995, pp 474-483

[46] Mallet E, Lanse X, Devaux AM, et al. Hypercalcitoninaemia in fulminant meningococcaemia in children. Lancet 1983; i:294

[47] Chesney RW, McCarron DM, Haddad JG, et al. Pathogenic mechanisms of the hypocalcemia of the staphylococcal toxic-shock syndrome. J Lab Clin Med 1983; 101:576-585

[48] Becker KL, Silva OL, Snider RH, et al. The pathophysiology of pulmonary calcitonin. In: The Endocrine Lung in Health and Disease. Becker KL, Gazdar AF (Eds). Philadelphia, WB Saunders, 1984, pp 277-279

[49] Lind L, Bucht E, Ljunghall S. Pronounced elevation in circulating calcitonin in critical care patients is related to the severity of illness and survival. Intensive Care Med 1995; 21:63-66

[50] Gendrel D, Assicot M, Raymond J, et al. Procalcitonin as a marker for the early diagnosis of neonatal infection. J Pediatr 1996; 128:570-573

[51] de Werra I, Jaccard C, Corradin SB. Cytokines, nitrite/nitrate, soluble tumor necrosis factor receptors, and procalcitonin concentrations: Comparisons in patients with septic shock, cardiogenic shock, and bacterial pneumonia. Crit Care Med 1997; 25:607-613

[52] Gérard Y, Hober D, Assicot M, et al. Procalcitonin as a marker of bacterial sepsis in patients infected with HIV-1. J Infect 1997; 35:41-46 
[53] Zazula R, Prucha M, Tyll T, et al. Induction of procalcitonin in liver transplant patients treated with anti-thymocyte globulin. Crit Care. 2007; 11(6):R131.

[54] Meissner M, Adina H, Schmidt J. Correlation of procalcitonin and C-reactiv protein to inflammation, complications and outcome during intensive care unit course of multiple-trauma patients. Critical Care; 2006;10;1;1-10

[55] Meisner M, Müller V, Khakpour Z, et al. Induction of procalcitonin and proinflammatory cytokines in an anhepatic baboon endotoxin shock model. Shock. 2003;19(2):187-90.

[56] Coelho MCM, Tannuri U, Tannuri ACA, et al.Is procalcitonin useful to differentiate rejection from bacterial infection in the early post-operative period of liver transplantation in children? Pediatr Transplantation 2009: 13: 1004-1006.

[57] Simon L, Gauvin F, Amre DK, et al. Serum procalcitonin and c-reactive protein levels as markers of bacterial infection: a systematic review and meta-analysis. Clin Infect Dis 2004;39:206-217.

[58] Cooper D, Sharples L, Cornelissen J, et al. Comparison between procalcitonin, serum amyloid A, and C-reactive protein as markers of serious bacterial and fungal infections after solid organ transplantation. Transplant Proc 2001;33: 1808-1810

[59] Fazakas J, Gondos T, Varga M, et al. Analysis of systemic and regional procalcitonin serum levels during liver transplantation. Transpl Int 2003: 16: 465-470.

[60] Kunz D, Pross M, Konig W, et al. Diagnostic relevance of procalcitonin, IL-6 and cellular immune status in the early phase after liver transplantation. Transplant Proc 1998;30:2398-2399

[61] Wiesner R, Edwards E, Freeman R, et al. United Network for Organ Sharing Liver Disease Severity Score Committee Model for end-stage liver disease (MELD) and allocation of donor livers.Gastroenterology.2003; 124(1):91-6.

[62] Dindo D, Demartines N, Clavien PA. Classification of surgical complications: a new proposal with evaluation in a cohort of 6336 patients and results of a survey. Ann Surg. 2004; 240(2):205-13.

[63] Deschênes M, Belle SH, Krom RA,et al. Early allograft dysfunction after liver transplantation: a definition and predictors of outcome. National Institute of Diabetes and Digestive and Kidney Diseases Liver Transplantation Database.Transplantation. 1998 15; 66(3):302-10.

[64] Calandra T, Cohen J. International Sepsis Forum Definition of Infection in the ICU Consensus Conference The international sepsis forum consensus conference on definitions of infection in the intensive care unit. Crit Care Med. 2005; 33(7):153848.

[65] BANFF schema for grading liver allograft rejection: An international consensus document. Hepatology 1997: 25: 658- 663.

[66] Dogan S, Aslan M. Hepatic ischemia-reperfusion injury and therapeutic strategies to alleviate cellular damage. Hepatol Res. 2011;41(2):103-17 
[67] Jaeschke H. Molecular mechanisms of hepatic ischemia reperfusion injury and preconditioning. Am J Physiol Gastrointest Liver Physiol 2003; 284:G15-G26. 